\title{
COM701 (A NOVEL IMMUNE CHECKPOINT INHIBITOR) IN PATIENTS WITH ADVANCED SOLID TUMORS.
}

\author{
A. ElNaggarr ${ }^{1}$, J. Luke ${ }^{\star 2}$, E. Hamilton ${ }^{3}$, B. Chmielowski ${ }^{4}$, E. lleana-Dumbrava ${ }^{5}$, A. Patnaik ${ }^{6}$, E. Lim \\ A.H Adewoye ${ }^{8}$, J. Hunter ${ }^{8}$, J. Olweny ${ }^{8}$, M. Sharma ${ }^{9}$, R. Sullivan ${ }^{10}$, D. Vaena ${ }^{1}$, D. Rasco ${ }^{6}$. \\ ${ }^{1}$ West Cancer Center and Research Institute., Gynecologic Oncology., Memphis, TN., USA. ${ }^{2}$ The University of Chicago Medical \\ Center., Medical Oncology., Chicago, IL., USA. 'Sarah Cannon Research Institute/Tennessee Oncology., Medical Oncology., \\ Nashville, TN., USA. ${ }^{4}$ University of California Los Angeles., Medical Oncology, Los Angeles, CA., USA. ${ }^{5}$ The University of Texas MD \\ Anderson Cancer Center., Department of Investigational Cancer Therapeutics., Houston, TX., USA. ${ }^{6}$ South Texas Accelerated \\ Research Therapeutics START., Medical Oncology., San Antonio, TX., USA. ${ }^{7}$ Columbia University., Medical Oncology., New York, \\ NY., USA. ${ }^{8}$ Compugen USA Inc., Clinical Development., South San Francisco, CA, USA. ${ }^{9}$ START Midwest., Medical Oncology., Grand \\ Rapids, MI., USA. ${ }^{10}$ Massachusetts General Hospital., Medical Oncology., Boston, MA., USA.
}

Objectives:

COM701 is a novel $1^{\text {st }}$ in-class monoclonal antibody (checkpoint inhibitor) of poliovirus receptor related immunoglobulin domain containing (PVRIG) blocking its interaction with it's ligand, PVRL2 (Fig 1). In preclinical experiments inhibition of PVRIG leads to activation of Tcells in the tumor microenvironment generating an antitumor immune response leading to tumor growth inhibition (Fig 2). We hypothesized that COM701 monotherapy and in combination with nivolumab will be safe and tolerable and demonstrate antitumor activity in pts with advanced solid tumors. Key primary objectives: safety and tolerability of COM701 monotherapy and in combination with nivolumab measured by the incidence of pts with adverse events and dose-limiting toxicities. The recommended dose for expansion of COM701 monotherapy and in combination with nivolumab. Key secondary objectives: the preliminary antitumor activity of COM701 monotherapy and with nivolumab in pts with endometrial, ovarian, breast and lung cancer. These tumor types were selected based on high expression of PVRL2.

Methods:

Study design: Dose escalation hybrid accelerated titration and $3+3$ design. Key inclusion criteria: Age $\geq 18$ yrs, advanced solid tumor and has exhausted all available standard therapy, prior checkpoint inhibitor permissible. Key exclusion criteria: history of immune-related events that led to immunotherapy treatment discontinuation. Expansion cohorts will enroll pts with the tumor types selected. ClinicalTrials.gov Identifier: NCT03667716.

Results:

No DLTs have been observed up to 7th COM701 monotherapy dose level and dose level 2 in Arm B (Fig 3).

Conclusions:

COM701 monotherapy and in combination with nivolumab has an acceptable safety and tolerable profile at the dose levels tested. Study enrollment is ongoing.
Fig 1.

PVRIG IS A Novel Checkpoint in the TIGIT/DNAM-1 AXIS
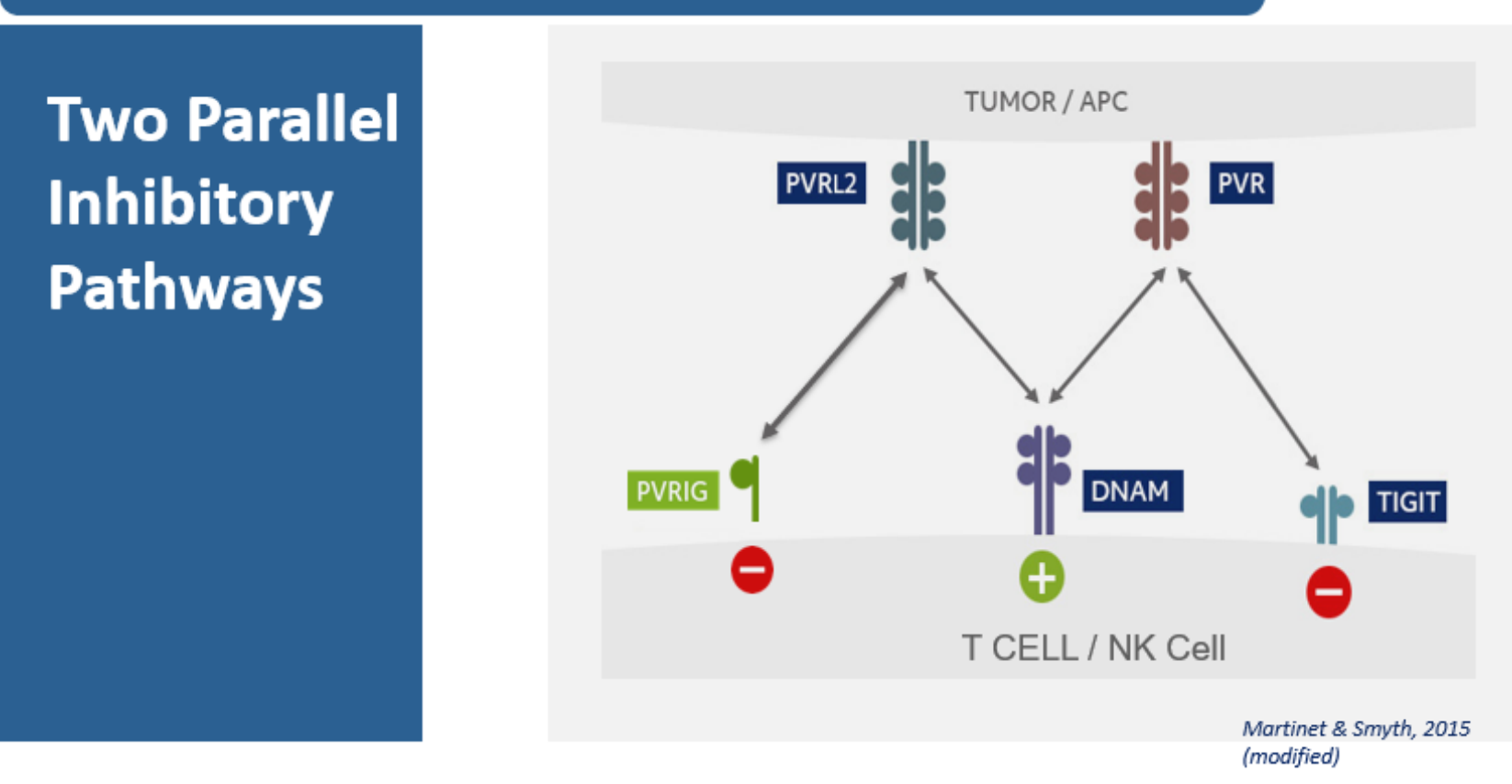

Fig 2.

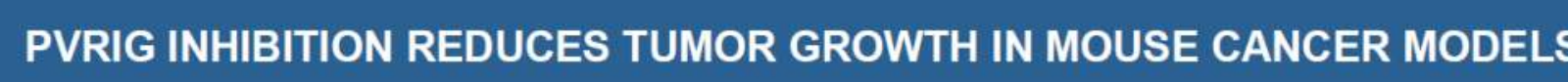

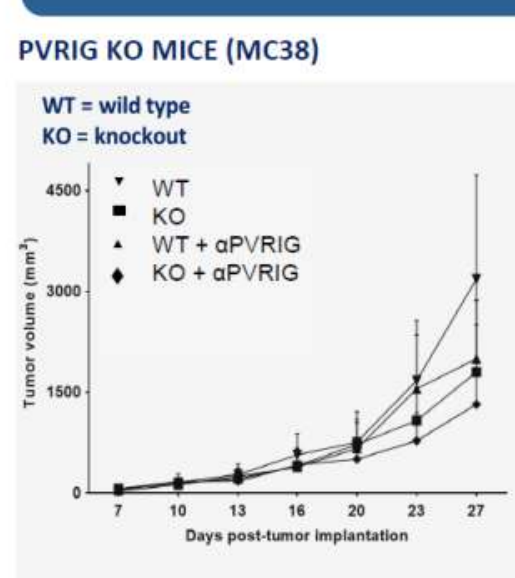

Reduced tumor growth in Ko mice

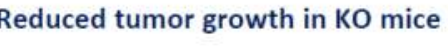

anti-PVRIG + TIGIT KO (B16)

anti-PVRIG + anti-PD1 (CT26)

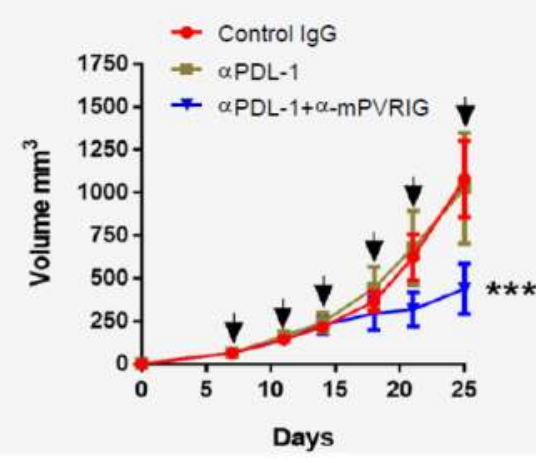

Synergistic tumor growth inhibition Synergistic tum
with anti-PD1

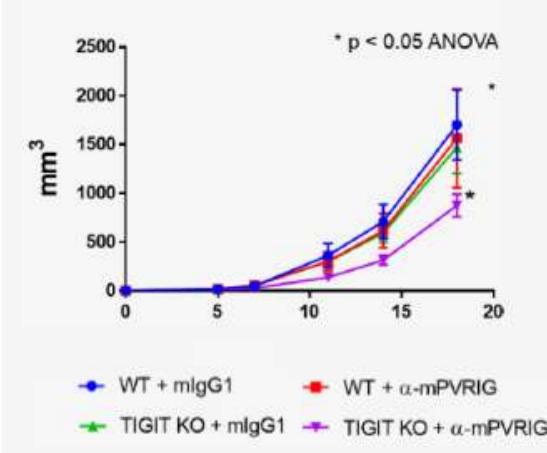

PVRIG inhibition required for tumor growth inhibition in TIGIT KO mice
Fig 3.

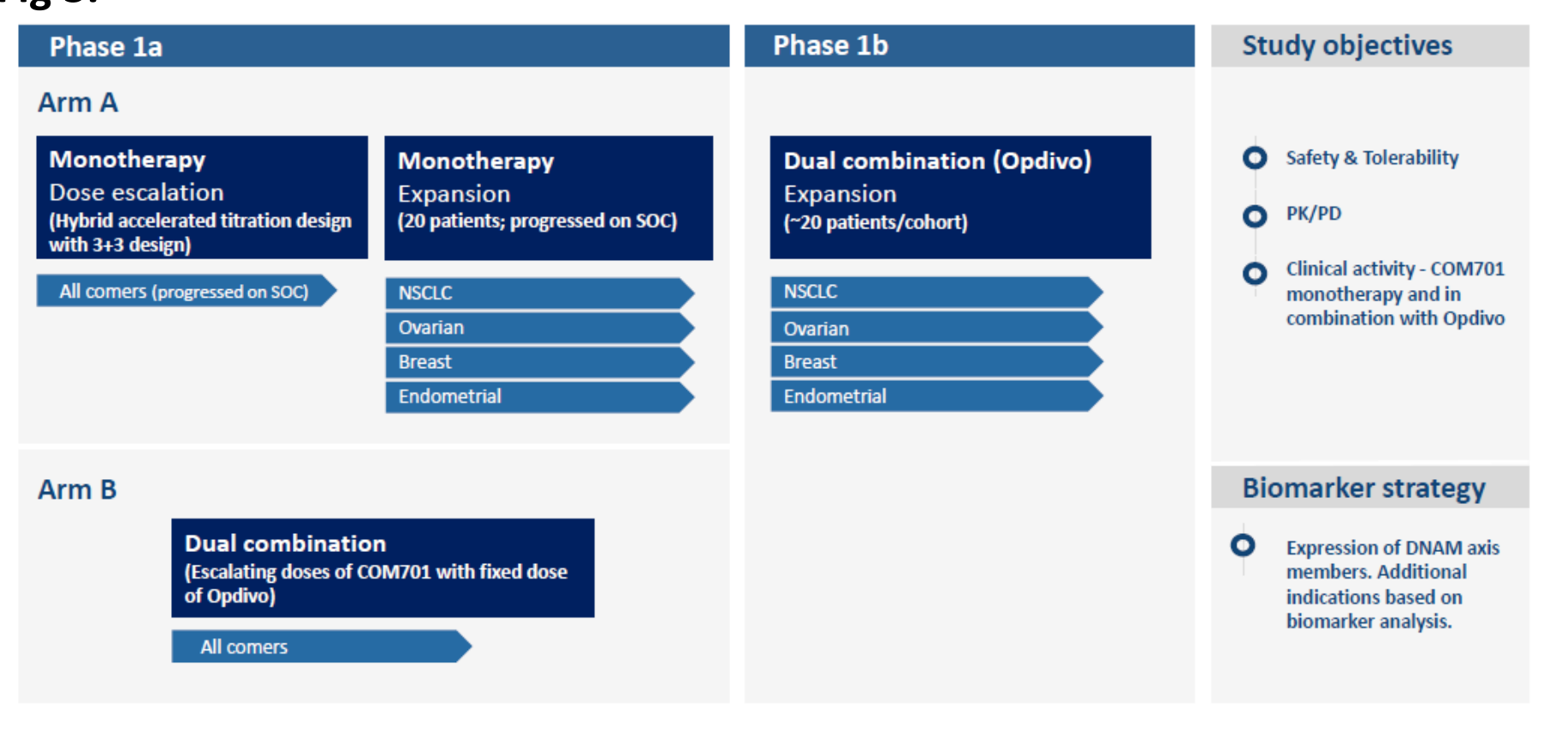

Acknowledgment: We thank the patients and their families, clinical trial investigators and their staff for participating in this clinical trial.

Study NCT03667716 is in collaboration with Bristol-Myers Squibb. 